\title{
Funciones Ejecutivas, Conductas Externalizantes e Internalizantes en Niñas y Adolescentes Expuestas y no Expuestas a un Entorno de Alta Adversidad Social ${ }^{\star 10}$
}

\author{
Eliana Ocampo Rojas \\ Psicóloga, Especialista en Evaluación y Diagnóstico \\ Neuropsicológico, Magíster en Neuropsicología \\ Universidad Pedagógica y Tecnológica de Colombia \\ Correo electrónico: eliana.ocampo@uptc.edu.co
}

Saby Saravia Santamaría

Psicóloga, Especialista en Evaluación y Diagnóstico Neuropsicológico, Magíster en Neuropsicología Correo electrónico: saby_s_s@hotmail.com

César Rey Anacona

Psicólogo, Doctor en Psicología Clínica y de la Salud Universidad Pedagógica y Tecnológica de Colombia Correo electrónico: cesar.rey@uptc.edu.co
Recibido: $18 / 01 / 2019$

Evaluado: 06/03/2019 Aceptado: 05/05/2019

\section{Resumen}

Los entornos de alta adversidad social como fue la "Zona del Bronx" en Bogotá (Colombia), se han relacionado con déficits ejecutivos y problemas comportamentales en niños y adolescentes. El objetivo de este estudio fue comparar las funciones ejecutivas (FFEE), conductas externalizantes e internalizantes entre niñas y adolescentes de 8 a 15 años que vivían en dicha zona, con niñas y adolescentes de las mismas edades y estratos socioeconómicos, mediante un diseño comparativotransversal. Se utilizaron las pruebas BANFE 2, BRIEF y CBCL. Los resultados señalan menos estrategias para controlar la impulsividad y más conductas de rompimiento de normas entre las participantes que habitaban dicha zona, aunque éstas presentaron puntuaciones significativamente más altas en las FFEE: cambio, monitoreo, iniciativa, planificación/organización y memoria de trabajo visoespacial, resultados que indican que la supervivencia en este tipo de entornos podría promover el desarrollo de estas funciones.

\section{Palabras clave}

Funciones ejecutivas, conductas externalizantes, conductas internalizantes, niñas, adolescentes.

10 Para citar este artículo: Ocampo, E., J., Saravia, S. \& Rey, C. (2020). Funciones Ejecutivas, Conductas Externalizantes e Internalizantes en Niñas y Adolescentes Expuestas y no Expuestas a un Entorno de Alta Adversidad Social. Informes Psicológicos, 20(1), pp. 147-166. http://dx.doi.org/10.18566/infpsic.v20n1a010

Este estudio se realizó dentro de la línea de investigación sobre trastornos del comportamiento de la Maestría en Neuropsicología Clínica de la Universidad de San Buenaventura Bogotá. 


\section{Executive functions, externalizing and internalizing behaviors in girls and adolescents exposed and not exposed to an environment of high social adversity}

Abstract

Environments of high social adversity such as the "Bronx Zone" in Bogotá (Colombia) have been related to executive deficits and behavioral problems in children and adolescents. The objective of this study was to compare the executive functions (FFEE) and the externalizing and internalizing behaviors among girls and adolescents aged 8 to 15 living in that area, with girls and adolescents of the same ages and socioeconomic strata, through a cross-comparative design. The BANFE 2, BRIEF and CBCL tests were used. The results indicate less strategies to control the impulsivity and more rule breaking behaviors among the participants that inhabited this area, although these presented significantly higher scores in the FFEE: change, monitoring, initiative, planning/organization and visospatial work memory, results that indicate that survival in these types of environments could promote the development of these functions.

Keywords

Xecutive functions, externalizing behaviors, internalizing behaviors, girls, adolescents.

\section{Funções executivas, comportamentos de externalização e internalização em meninas e adolescentes expostas e não expostas a um ambiente de alta adversidade social}

Resumo

Os ambientes de alta adversidade social, como a "Zona do Bronx" em Bogotá (Colômbia), têm sido relacionados a déficits executivos e problemas comportamentais em crianças e adolescentes. 0 objetivo deste estudo foi comparar funções executivas (FFEE), comportamentos externalizantes e internalizantes entre meninas e adolescentes de 8 a 15 anos residentes nessa área, com meninas e adolescentes da mesma idade e estratos socioeconômicos, por meio de um desenho comparativo-transversal. Foram utilizados os testes BANFE 2, BREVE e CBCL. Os resultados indicam menos estratégias para controlar a impulsividade e mais comportamento de quebra de regras entre os participantes que habitaram essa área, embora estes tenham apresentado escores significativamente mais altos na FFEE: mudança, monitoramento, iniciativa, planejamento / organização e memória do trabalho visuoespacial, resultados que indicam que a sobrevivência nesses tipos de ambientes pode promover o desenvolvimento dessas funções.

Palavras chave

Unções executivas, comportamentos de externalização, comportamentos de internalização, meninas, adolescentes. 


\section{ntroducción}

Las funciones ejecutivas (FFEE) hacen referencia a una serie de procesos orientados hacia la resolución de situaciones complejas, mediante la formulación de metas, la planificación de los procesos y la regulación de la conducta, la motivación y las emociones (Cabas, González \& Mendoza, 2018; Flores-Lázaro, CastilloPreciado \& Jiménez-Miramonte, 2014; Tirapu-Ustárroz, García-Molina, Luna-Lario, Roig-Rovira \& Pelegrín-Valero, 2008; Tirapu, García, Luna, Verdejo \& Ríos, 2012). Se considera que el funcionamiento ejecutivo depende fundamentalmente de la corteza prefrontal, por lo que las alteraciones en el desarrollo de esta estructura desde temprana edad pueden influir en el desarrollo de las FFEE (Flores \& OstroskyShejet, 2012).

La evidencia disponible señala que las personas que crecen en ambientes altamente estresantes, como los entornos de adversidad social por situaciones de pobreza, violencia intrafamiliar y delincuencia, tienden a puntuar más bajo en pruebas de funciones ejecutivas, de inteligencia, lenguaje, memoria y otras habilidades cognitivas, alterando su rendimiento académico y fomentando los problemas de comportamiento (Barrasso-Catanzaro \& Eslinger, 2016; Dike, 2017; Hostinar, Stellern, Schaefer, Carlson \& Gunnar, 2012; Mani, Mullainathan, Shafir \& Zhao, 2013). La deprivación en edades tempranas también se ha relacionado con déficits en funciones ejecutivas y problemas de comportamiento infantil (Barrasso-Catanzaro \& Eslinger, 2016; Troller, Nelson, Zeanah \& Fox, 2016). Madhushanthi, Wimalasekera, Goonewardena, Amarasekara y Lenora (2018), por ejemplo, encontraron puntuaciones más bajas en memoria de trabajo e inhibición en las participantes de estrato socioeconómico bajo, comparadas con las de estrato medio, en una muestra de 200 adolescentes mujeres de 11 a 14 años de Sri Lanka.

En relación con los problemas de comportamiento infantil, Achenbach y Edelbrock (1992) identificaron una serie de síndromes que podrían categorizarse conforme a dos dimensiones básicas: internalizantes y externalizantes. Las conductas "internalizantes", incluyen síntomas de ansiedad, depresión, retraimiento, aislamiento, temor y quejas somáticas y tienen que ver con aquellas dificultades que afectan directamente al mismo individuo, mientras que las conductas "externalizantes", involucran los síntomas propios de los trastornos de conducta y otros relacionados, como agresividad, desobediencia, crueldad hacia los animales, destructividad, abuso de sustancias e hiperactividad, y se caracterizan porque suelen crear problemas a las personas que los rodean, siendo más visibles en el contexto familiar y escolar (Moreno \& Utria, 2011) .

La evidencia indica que las conductas de tipo internalizante son más comunes entre las niñas y las adolescentes, mientras que las externalizantes son más frecuentes entre los varones (Hewitt, 2015). En un estudio en el que se compararon por sexo las conductas internalizantes y externalizantes reportadas por 298 estudiantes peruanos de 12 a 18 años de edad, por ejemplo, se encontró que las mujeres puntuaban significativamente más alto en ansiedad, depresión, quejas somáticas, problemas atencionales y conductas internalizantes, en general, mientras que los hombres puntuaron más alto en 
rompimiento de reglas y conductas externalizantes (Alarcón \& Bárrig, 2015).

Los déficits en FFEE se han relacionado con problemas de comportamiento externalizante e internalizante desde la primera infancia (Hatoum, Rhee, Corley, Hewitt \& Friedman, 2018). Quistberg y Mueller (2019), por ejemplo, encontraron que los errores en el control inhibitorio se relacionaban con las conductas externalizantes, mientras que los errores en la memoria de trabajo se asociaban con las conductas internalizantes entre 69 niños de 5 y 6 años de edad.

Según Lezama (2015), los déficits en FFEE podrían relacionarse con las conductas externalizantes en la infancia, ya que estas funciones le permiten al individuo regular su comportamiento y sus emociones, reduciendo la impulsividad, mientras que los niños con un desarrollo normal en este tipo de funciones tienden a comportarse de una forma más prosocial, son más competentes socialmente y presentan menos problemas de conducta. Andrés et al. (2010), en ese sentido, encontraron un desempeño más bajo en FFEE entre los niños que asistían a escuelas públicas y que obtuvieron una puntuación alta en conductas antisociales conforme a la Lista de Verificación de Conducta Infantil (CBCL; Achenbach \& Edelbrock, 1992), en una muestra conformada por 65 niños argentinos de 6 a 8 años de edad.

Woltering, Lishak, Hodgson, Granic y Zelazo (2016), por su parte, compararon a un grupo de 93 niños y niñas entre los 7 y 12 años de edad, que puntuaron alto en el índice de conductas externalizantes y/o internalizantes de la CBCL (Achenbach, 1991), con un grupo de 63 niños y niñas sin dificultades en el desarrollo, en varias medidas de FFEE frías y calientes. Los niños(as) del primer grupo puntuaron significativamente más bajo en las pruebas de FFEE calientes, mas no en las frías, comparados con los niños(as) del otro grupo. Además, presentaron una mayor reactividad emocional ante estímulos con matiz emocional, particularmente los participantes que tenían tanto síntomas internalizantes como externalizantes, comparados con los que solo presentaban síntomas externalizantes.

Otros estudios muestran que las condiciones de vida adversas pueden relacionarse con problemas de comportamiento externalizante e internalizante. Boutwell, Helton, Vaughn y Kavish (2017), por ejemplo, examinaron la relación entre los problemas de comportamiento internalizante y externalizante y el coeficiente intelectual (Cl) entre 2591 niños estadounidenses cuyas familias estaban siendo investigadas por denuncias de maltrato, encontrando una relación estadísticamente significativa entre un bajo $\mathrm{Cl}$ y puntajes más altos en problemas de conducta de los dos tipos, lo que sugiere que la situación de maltrato podría asociarse con un bajo $\mathrm{Cl}$, incrementando los problemas de comportamiento de los dos tipos. Kersten et al. (2017) por su parte, encontraron en una muestra de 1178 niños y adolescentes estadounidenses de 9 a 18 años, una asociación estadísticamente significativa entre ser testigo de violencia en la comunidad y problemas de conducta, tanto en los individuos normales de dicha muestra como en aquellos que presentaban trastorno de conducta, relación que se mantuvo estable independientemente del género, la edad y el estrato socioeconómico. 
En conjunto, esta evidencia señala que los niños y adolescentes que crecen en condiciones de adversidad social, debido a la pobreza, el abandono, la negligencia, el descuido o los malos tratos físicos, psicológicos y sexuales, están en un riesgo mayor de presentar alteraciones en su desarrollo cognitivo y ejecutivo, promoviendo la aparición de problemas de comportamiento internalizante y externalizante, al afectar la capacidad de regulación conductual y emocional.

No obstante, otros autores plantean una perspectiva diametralmente opuesta con respecto a los posibles efectos de este tipo de entornos, señalando que la exposición crónica a los mismos podría promover el desarrollo de algunas funciones cognitivas y ejecutivas, necesarias para sobrevivir en los mismos (Del Giudice, Hinnant, Ellis \& El-Sheikh, 2012; Ellis \& Del Giudice, 2014; Ellis et al., 2012; Frankenhuis \& Del Giudice, 2012; Nederhof \& Schmidt, 2012). Mittal, Griskevicius, Simpson, Sung y Young (2015), por ejemplo, encontraron puntuaciones más altas en la función ejecutiva de cambio, entre adultos que crecieron en entornos violentos e impredecibles comparados con adultos que no crecieron en este tipo de ambientes, aunque presentaron una menor capacidad de inhibición que estos últimos, resaltando que este tipo de entornos exige que los niños desarrollen la habilidad para cambiar rápidamente el foco atencional para sobrevivir en tales entornos.

En Colombia, miles de niños, niñas y adolescentes viven en entornos de alta adversidad social por situaciones de pobreza, violencia social, violencia intrafamiliar y delincuencia, haciéndolos proclives a presentar déficits en su funcionamiento ejecutivo y problemas de comportamiento externalizante e internalizante. Sin embargo, no se cuenta con estudios sobre el funcionamiento ejecutivo y este tipo de conductas en esta población. Moreno, Echavarría, Pardo y Quiñones (2014) encontraron entre 63 adolescentes varones y mujeres de 12 a 16 años de edad, de un colegio público de Bogotá (Colombia), que el $19.56 \%$ presentaba conductas internalizantes, mientras que Hewitt (2015) halló una frecuencia significativamente mayor de conductas internalizantes y externalizantes, medidas con la CBCL, entre 452 niños y niñas de 8 a 12 años de edad de colegios de estratos bajos, comparados con 452 niños y niñas del mismo rango de edades, vinculados a colegios de estratos medios, de la misma ciudad. Sin embargo, estos estudios se llevaron a cabo con muestras escolarizadas, sin tener en cuenta si estos participantes estaban expuestos o no a condiciones de alta adversidad social, analizándose solamente las conductas externalizantes e internalizantes.

En el centro de Bogotá (Colombia) recientemente se allanó un sector de alta peligrosidad conocido como la "Zona del Bronx", caracterizado por la venta y consumo de sustancias psicoactivas, la delincuencia, la indigencia, la prostitución y la pobreza entre muchos de sus residentes. Según Orozco (2016), muchos menores de edad asistían a ese lugar para consumir estas sustancias o para escapar de situaciones de violencia intrafamiliar, relacionándose así con la deserción escolar y las fugas del hogar. Este lugar se identificaba como una zona de miedo, símbolo de contradicciones y contrastes sociales y el principal expendio de drogas de la ciudad (Bravo, 2015). 
Teniendo en cuenta las consideraciones teóricas y empíricas expuestas anteriormente, es posible que las niñas y adolescentes que vivían en ese lugar, presentasen déficits en FFEE, así como un número mayor de comportamientos internalizantes y externalizantes, por lo que su estudio podría representar un punto de partida para incrementar el conocimiento sobre este tema en Colombia, generando alternativas basadas en la evidencia para la evaluación y tratamiento de esta población.

Con base en lo anterior, este trabajo se formuló como pregunta de investigación: ¿Existen diferencias en el funcionamiento ejecutivo, conductas externalizantes e internalizantes entre niñas y adolescentes de 8 a 15 años de edad, que vivían en la "Zona del Bronx", comparadas con niñas y adolescentes con características sociodemográficas similares de la misma ciudad?, planteándose como objetivo comparar el funcionamiento ejecutivo, las conductas externalizantes e internalizantes entre ambos grupos de niñas y adolescentes.

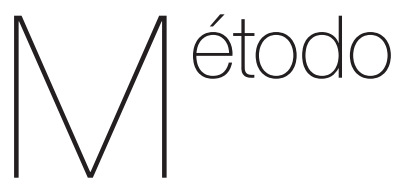

\section{Diseño}

El estudio se realizó desde un enfoque empírico analítico, mediante un diseño no experimental, descriptivo, transversal y comparativo. Descriptivo porque se efectuó una medición de un conjunto de variables sin modificarlas intencionalmente. Transversal porque solamente se realizó una medición de estas variables y comparativo porque se pretendía contraponer los datos arrojados por dos grupos de participantes (Rodríguez-Sabiote, 2016).

\section{Participantes}

En total participaron 27 niñas y adolescentes entre 8 y 15 años de edad $(X=$ 12.85; $D X=2.49$ ), de estratos socioeconómicos uno y dos, que vivían en la ciudad de Bogotá. Las niñas y adolescentes que vivían en un entorno de alta adversidad social $(n=16)$ tenían entre 10 y 15 años de edad $(X=14.19 ; D X=1.72)$ y fueron seleccionadas de manera no aleatoria, según su disponibilidad, entre los niños, niñas y adolescentes que se encontraban internadas en el marco de un proceso de restablecimiento de derechos, por parte del Instituto Colombiano de Bienestar Familiar (ICBF), Regional Bogotá, debido a que fueron rescatados después del allanamiento policial del sector de tráfico de drogas conocido como "Zona del Bronx", en mayo de 2016. Estas niñas y adolescentes habían cursado una media de 5.31 grados escolares $(D X=1.35)$.

La niñas y adolescentes del grupo de comparación ( $n=16)$, tenían entre 8 y 15 años de edad $(X=10.91 ; D X=2.17)$ y también fueron seleccionadas de forma no aleatoria, según su disponibilidad, en un colegio distrital del sur de Bogotá, cuyos estudiantes habitan barrios de estratos bajos (uno y dos). Estas niñas y adolescentes habían cursado un promedio de 5.09 grados escolares $(D X=1.51)$.

Los criterios de inclusión y exclusión de los dos grupos fueron: (a) tener una edad ubicada en los años escolares o la adolescencia, (b) no tener discapacidad cognitiva, conforme a los resultados 
obtenidos en el Test breve de Inteligencia de Kaufman (Kaufman \& Kaufman, 2000) y (c) no presentar antecedentes de patologías neurológicas o físicas graves según los registros que tenía de cada participante en la institución correspondiente.

\section{Instrumentos}

Test breve de Inteligencia de Kaufman (K-BIT; Kaufman \& Kaufman, 2000). Es un test de "screening" que permite llegar a una rápida apreciación de la inteligencia general del niño o adolescente mediante dos subtest: (a) Vocabulario, que a su vez consta de dos pruebas: vocabulario expresivo y definiciones, las cuales miden las habilidades verbales relacionadas con el aprendizaje escolar y (b) subtest de Matrices, que evalúa la capacidad para resolver problemas de razonamiento. Suministra un C.I. verbal, un C.I. no verbal y un C.I. compuesto que resume el rendimiento total en el test. Los autores reportan coeficientes de fiabilidad que varían según el rango de edad, superiores a .76. El C.l. compuesto presentó una alta correlación (.63) con las puntuaciones de la prueba K-ABC y el C.I. compuesto de la prueba WAIS-R (.75), datos a favor de la validez del instrumento (Kaufman \& Kaufman, 2000). Este test no ha sido validado con muestras colombianas.

\footnotetext{
Batería Neuropsicológica de Funciones Ejecutivas y Lóbulos Frontales (BANFE 2; Flores, Ostrosky \& Lozano, 2014). Esta batería agrupa un número importante de pruebas neuropsicológicas reconocidas internacionalmente en la evaluación de las funciones que dependen principalmente de la corteza prefrontal. Estas pruebas presentan una alta especificidad de área, determinada por estudios
}

en individuos con daño cerebral y estudios de neuroimagen funcional, las cuales se seleccionaron y dividieron principalmente con base en el criterio anatómicofuncional, evaluando funciones que dependen de la corteza orbito-frontal (COF), la corteza prefrontal medial (CPFM), la corteza prefrontal dorsolateral (CPFDL) y la corteza prefrontal anterior (CPFA). Además de las pruebas específicas, la batería permite obtener un índice del funcionamiento de las tres áreas prefrontales evaluadas (COF-CPFM, CPFDL y CPFA) y un índice de desempeño global. Requiere de un tiempo aproximado de aplicación de 50 minutos, el cual depende de la edad, la escolaridad y/o el trastorno neurológico o psicológico por el cual la persona solicita la evaluación. En algunas tareas se toma el tiempo de ejecución y en otras no hay un tiempo límite para concluirlas. Este test no ha sido validado con muestras colombianas.

\section{Evaluación Conductual de la Fun-} ción Ejecutiva (BRIEF; Gioia, Isquith, Guy \& Kenworthy, 2000). Es una prueba de referencia a nivel internacional para la evaluación de los aspectos más cotidianos y conductuales de las FFEE por parte de padres y profesores de niños y adolescentes entre 5 y 18 años de edad. Dispone de dos formas (BRIEF-Familia y BRIEF-Escuela) que pueden aplicarse por separado o conjuntamente, cuyos 86 ítems preguntan por la frecuencia con que aparecen una serie de conductas a través de una escala Likert de tres opciones de respuesta ("Nunca": 0; "Algunas veces": 1 y "Siempre": 2). La aplicación de la prueba puede ser individual o colectiva, en un tiempo entre 10 y 15 minutos aproximadamente. 
Este instrumento presenta los siguientes índices: (a) Índice de Regulación del Comportamiento, que incluye las escalas de Inhibición, Cambio y Control Emocional; (b) Índice de Metacognición, que abarca las escalas de Iniciativa, Memoria de Trabajo, Organización/Planificación, Organización de Materiales y Monitoreo y (c) el Índice Global de Función Ejecutiva, que consiste en la sumatoria de los dos índices anteriores. Los autores reportan índices de consistencia interna que varían entre .85 y .96 para las escalas, .93 para el Índice de Regulación del Comportamiento y el Índice de Metacognición y .96 para el Índice Global. La fiabilidad test-retest que obtuvieron fue .82, encontrando correlaciones estadísticamente significativas con medidas de inatención, impulsividad y de aprendizaje, lo que indica una buena validez convergente (Gioia et al., 2000). Este test no ha sido validado con muestras colombianas. En este estudio, la consistencia interna obtenida a través de la prueba Alfa de Cronbach fue de .98.

Lista de Verificación de Conducta Infantil (CBCL; Achenbach \& Edelbrock, 1992). Es una lista de conductas que ofrece un perfil de los problemas individuales de los niños y adolescentes entre 6 y 18 años, a partir del reporte de los padres/cuidadores y/o de los maestros, los cuales se incluyen en seis síndromes clasificados de acuerdo con su carácter internalizante o externalizante, así: (a) internalizantes: ansioso depresivo, retraído depresivo y quejas somáticas, (b) mixtos: problemas sociales, problemas de pensamiento y problemas de atención y (c) externalizantes: rompimiento de normas y conducta agresiva. Fue validada con una muestra de 1531 padres de niños y adolescentes colombianos de 6 a 18 años, encontrándose una consistencia interna de .94. El análisis factorial confirmatorio demostró un buen ajuste de los datos y una estructura factorial acorde con las dimensiones teóricas propuestas y planteadas por los autores del instrumento (Hewitt, Jaimes, Vera \& Villa, 2012). En esta investigación, la consistencia interna obtenida a través de la prueba Alfa de Cronbach fue de .96.

\section{Procedimiento}

Inicialmente se obtuvieron los permisos institucionales para contactar a los cuidadores del grupo de niñas y niñas resguardadas por el ICBF (Vg., supervisores de contrato y defensores de familia), así como a los padres de familia del grupo de comparación, seleccionándose aquellas niñas y adolescentes que estuvieron dentro del rango de edades deseado y que no presentaran patologías neurológicas o físicas graves según los registros de cada institución. Posteriormente se aplicó la CBCL a dichos padres o cuidadores, leyendo los ítems a los padres que no supieran leer y escribir, la BRIEF a los profesores de las niñas y adolescentes seleccionadas y las pruebas K-BIT y BANFE 2 a estas últimas. La aplicación de estas dos últimas pruebas duró aproximadamente hora y media, efectuándose de manera individual en un lugar con adecuadas condiciones ambientales, verificando previamente que cada niña o adolescente estuviera en adecuadas condiciones físicas y anímicas. Se descartó la participación de ocho menores de edad debido a que obtuvieron una puntuación igual o inferior a 70 o en el K-BIT. 
Los datos obtenidos se incorporaron en una base de datos de programa SPSS versión 22.0, calculando inicialmente las medias y las desviaciones estándar, aplicándose posteriormente la prueba de normalidad Shapiro-Wilk, debido al número de observaciones (menos de 50), encontrándose que la mayoría de las variables no se distribuía de manera normal, por lo que se decidió aplicar la prueba U de Mann Whitney, para comparar los grupos y la prueba no paramétrica del tamaño del efecto para dicha prueba $(r=Z / \sqrt{ } N)$, para lo cual se tuvo en cuenta la clasificación de los tamaños del efecto propuestos por Cohen: (a) bajos: entre .1 y .3, (b) moderados: entre .4 y .7 y (c) altos: a partir de .8. No se calcularon los subtotales y la puntuación total de la BANFE 2, debido a que se contaba con baremos colombianos.

\section{Consideraciones éticas}

Los procedimientos de este estudio tuvieron en cuenta lo establecido en la Resolución 8430 de 1993 del Ministerio de Salud ("Normas científicas, técnicas y administrativas para la investigación en salud"), que establece en sus títulos I y II capítulos I y III, los aspectos éticos de la investigación en seres humanos y en los menores de edad o discapacidad, en particular. Con base en esta reglamentación, esta investigación se consideró de riesgo mínimo, categoría correspondiente a estudios prospectivos que emplean el registro de datos a través de procedimientos comunes consistentes en exámenes físicos o psicológicos de diagnóstico o tratamientos rutinarios. Los padres de familia y/o cuidadores y los defensores de familias y supervisores de contrato, según correspondiera, brindaron su consentimiento informado por escrito para la participación de las niñas y adolescentes en el estudio, así como para la publicación y divulgación de sus resultados con fines académicos, asegurando la debida confidencialidad de la información para proteger la identidad de los menores de edad. Este estudio también tuvo en cuenta los parámetros éticos establecidos en el código deontológico del psicólogo, establecido en la Ley 1090 de 2006, referentes a la investigación con seres humanos, el principio de confidencialidad y la administración de pruebas psicológicas.

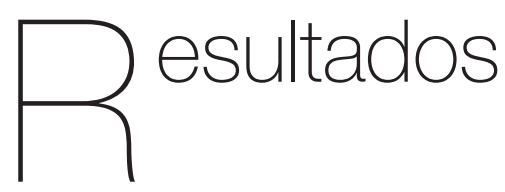

A continuación, se presentan los resultados de las comparaciones realizadas entre los dos grupos en las escalas y los índices de las pruebas utilizadas. En la Tabla 1 se presentan los resultados de las comparaciones realizadas con las escalas de la BRIEF, que muestran que el grupo que vivía en un entorno de alta adversidad social (GA) presentó rangos de puntuaciones significativamente más bajos en Planificación/Organización, aunque con un tamaño del efecto bajo (entre .1 y .3), así como en las escalas de Cambio, Iniciativa y Monitoreo y el Índice Global de Función Ejecutiva, con tamaños del efecto moderados (entre .4 y .7), que el grupo de comparación (GC). 
Tabla 1

Resultados de las comparaciones entre los grupos en las escalas e índices del BRIEF.

\begin{tabular}{|c|c|c|c|c|c|c|}
\hline Variable & Grupo & $\mathrm{n}$ & Rango & U & $\mathrm{p}$ & $r$ \\
\hline \multirow[t]{2}{*}{ Inhibición } & ICBF & 16 & 11.75 & \multirow{2}{*}{52.000} & \multirow{2}{*}{.074} & -.34 \\
\hline & Colegio & 11 & 17.27 & & & \\
\hline \multirow{2}{*}{ Cambio } & ICBF & 16 & 11.34 & \multirow{2}{*}{45.500} & \multirow{2}{*}{$.035^{*}$} & \multirow{2}{*}{-.41} \\
\hline & Colegio & 11 & 17.86 & & & \\
\hline \multirow{2}{*}{ Control emocional } & ICBF & 16 & 11.91 & \multirow{2}{*}{54.500} & \multirow{2}{*}{.097} & \multirow{2}{*}{-.32} \\
\hline & Colegio & 11 & 17.05 & & & \\
\hline \multirow{2}{*}{ Iniciativa } & ICBF & 16 & 11.00 & \multirow{2}{*}{40.000} & \multirow{2}{*}{$.017^{\star}$} & \multirow{2}{*}{-.46} \\
\hline & Colegio & 11 & 18.36 & & & \\
\hline \multirow{2}{*}{ Memoria de trabajo } & ICBF & 16 & 12.13 & \multirow{2}{*}{58.000} & \multirow{2}{*}{.138} & \multirow{2}{*}{-.29} \\
\hline & Colegio & 11 & 16.73 & & & \\
\hline \multirow{2}{*}{ Planificación/ organización } & ICBF & 16 & 11.16 & \multirow{2}{*}{42.500} & \multirow{2}{*}{$.024^{*}$} & \multirow{2}{*}{.024} \\
\hline & Colegio & 11 & 18.14 & & & \\
\hline \multirow{2}{*}{ Organización de materiales } & ICBF & 16 & 12.78 & \multirow{2}{*}{68.500} & \multirow{2}{*}{.333} & \multirow{2}{*}{-.19} \\
\hline & Colegio & 11 & 15.77 & & & \\
\hline \multirow{2}{*}{ Monitoreo } & ICBF & 16 & 11.34 & \multirow{2}{*}{45.500} & \multirow{2}{*}{$.035^{*}$} & \multirow{2}{*}{-.40} \\
\hline & Colegio & 11 & 17.86 & & & \\
\hline \multirow{2}{*}{ Índice de regulación del comportamiento } & ICBF & 16 & 11.69 & \multirow{2}{*}{51.000} & \multirow{2}{*}{.068} & \\
\hline & Colegio & 11 & 17.36 & & & -.00 \\
\hline & ICBF & 16 & 11.56 & & & \\
\hline inaice de metacognicion & Colegio & 16 & 17.55 & 49.000 & .054 & -.31 \\
\hline 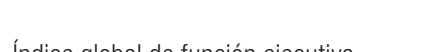 & ICBF & 11 & 11.50 & (20) & 0 & \\
\hline 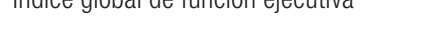 & Colegio & 16 & 17.64 & 40.000 & .040 & -.00 \\
\hline
\end{tabular}

Nota. $n$ : Número de participantes; U: Resultado de la U de Mann Whitney; $p$ : Probabilidad; $r$ : Tamaño del efecto no paramétrico; ${ }^{*} p \leq .05$

En la Tabla 2 se presentan los resultados de las comparaciones efectuadas con la BANFE 2, que indican que el GA presentó rangos de puntuaciones significativamente más altas en "Clasificación semántica Categorías Abstractas", "Resta consecutiva A Tiempo", "Orden alfabético Ensayo 3", "Memoria de trabajo Visoespacial", "Clasificación de cartas Perseveraciones", "Clasificación de cartas Perseveraciones diferidas", "Clasificación semántica Total categorías" y
"Clasificación semántica Puntaje total", con tamaños del efecto moderados, así como en "Laberintos Tiempo", con un tamaño del efecto alto. Por su parte, el GC obtuvo un rango promedio de puntuaciones significativamente más altas en "Stroop forma A errores tipo Stroop", "Clasificación de cartas errores de mantenimiento", "Resta consecutiva A Tiempo", "Suma consecutiva Tiempo" y “Laberintos Planeación", con tamaños del efecto moderados. 
Tabla 2

Resultados de las comparaciones entre los grupos en las pruebas de la BANFE.

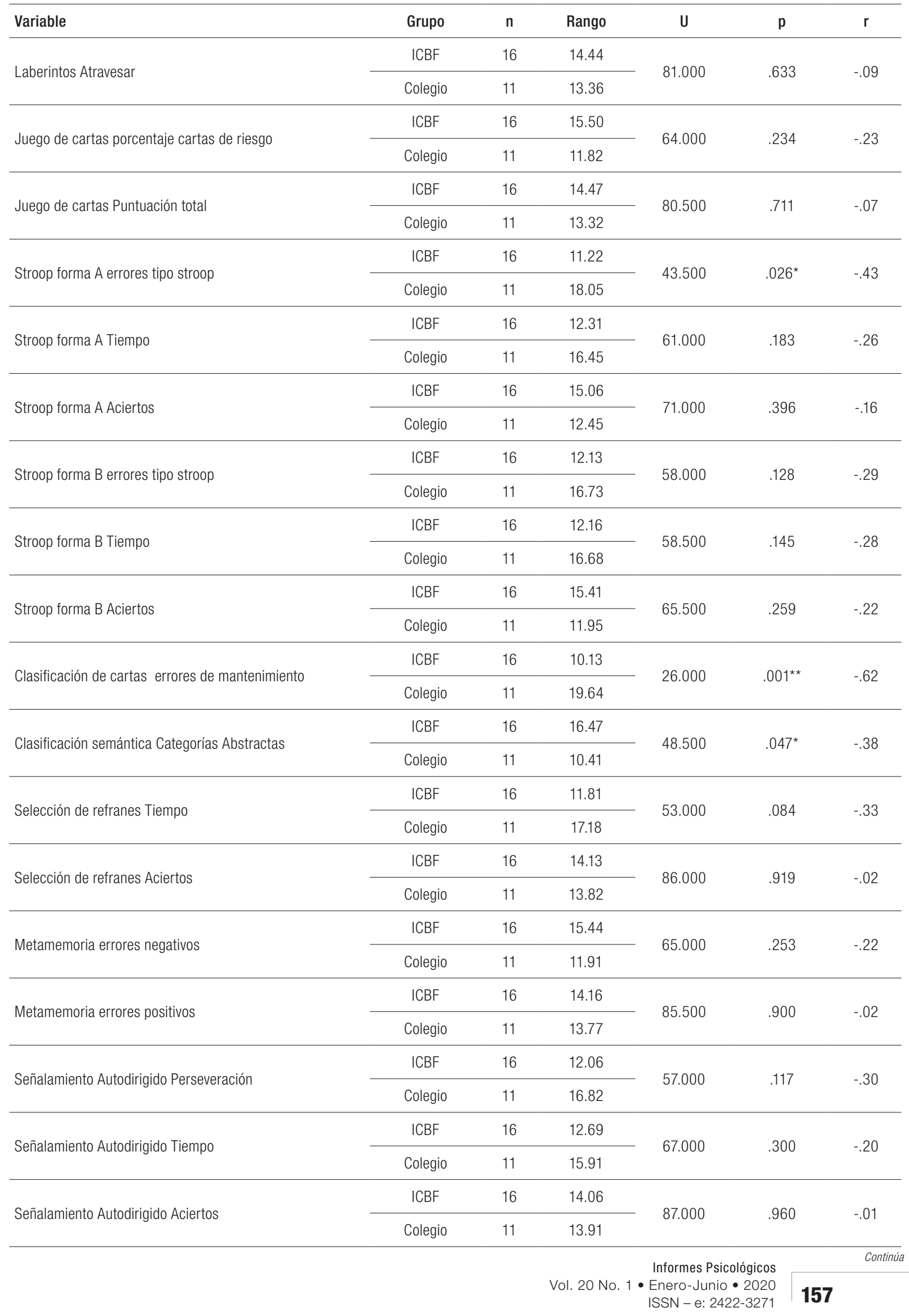


Continuación

\begin{tabular}{|c|c|c|c|c|c|c|}
\hline Variable & Grupo & $\mathrm{n}$ & Rango & U & $\mathrm{p}$ & $r$ \\
\hline \multirow{2}{*}{ Resta consecutiva A Tiempo } & ICBF & 16 & 9.81 & \multirow{2}{*}{21.000} & \multirow{2}{*}{$.001^{\star *}$} & \multirow{2}{*}{-.64} \\
\hline & Colegio & 11 & 20.09 & & & \\
\hline \multirow{2}{*}{ Resta consecutiva A Aciertos } & ICBF & 16 & 18.38 & \multirow{2}{*}{18.000} & \multirow{2}{*}{$.000^{* * *}$} & \multirow{2}{*}{-.72} \\
\hline & Colegio & 11 & 7.64 & & & \\
\hline \multirow{2}{*}{ Resta consecutiva B Tiempo } & ICBF & 16 & 10.69 & \multirow{2}{*}{35.000} & \multirow{2}{*}{.070} & \multirow{2}{*}{-.37} \\
\hline & Colegio & 8 & 16.13 & & & \\
\hline \multirow{2}{*}{ Resta consecutiva B Aciertos } & ICBF & 16 & 14.59 & \multirow{2}{*}{30.500} & \multirow{2}{*}{$.025^{\star}$} & \multirow{2}{*}{-.46} \\
\hline & Colegio & 8 & 8.31 & & & \\
\hline \multirow{2}{*}{ Suma consecutiva Tiempo } & $\mathrm{ICBF}$ & 16 & 11.47 & \multirow{2}{*}{47.500} & \multirow{2}{*}{$.046^{*}$} & \multirow{2}{*}{-.38} \\
\hline & Colegio & 11 & 17.68 & & & \\
\hline \multirow{2}{*}{ Suma consecutiva Aciertos } & ICBF & 16 & 15.56 & \multirow{2}{*}{63.000} & \multirow{2}{*}{.126} & \multirow{2}{*}{-.29} \\
\hline & Colegio & 11 & 11.73 & & & \\
\hline \multirow{2}{*}{ Orden alfabético Ensayo 1} & ICBF & 16 & 14.00 & \multirow{2}{*}{88.000} & \multirow{2}{*}{1.000} & \\
\hline & Colegio & 11 & 14.00 & & & .00 \\
\hline & ICBF & 16 & 16.13 & & & \\
\hline 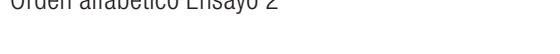 & Colegio & 11 & 10.91 & 54.000 & .084 & -.33 \\
\hline & ICBF & 16 & 17.16 & & & \\
\hline Orden alfabético Ensayo 3 & Colegio & 11 & 9.41 & 37.500 & $.010^{\star \star}$ & -.49 \\
\hline Aomoria do trahain Vliconcnacial Socuncia máximp & ICBF & 16 & 18.72 & 12500 & $0 \cap 0 * * * *$ & 78 \\
\hline Miemorla de trabajo visoespaclal secuencla maxıma & Colegio & 11 & 7.14 & 12.500 & $.000^{\operatorname{man}}$ & -.18 \\
\hline 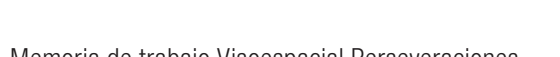 & ICBF & 16 & 13.88 & 的 & 800 & 01 \\
\hline 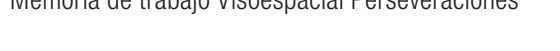 & Colegio & 11 & 14.18 & 86.000 & .828 & .04 \\
\hline & ICBF & 16 & 13.06 & & & \\
\hline Memoria de trabajo Visoespacial Errores de orden & Colegio & 11 & 15.36 & 73.000 & .456 & -.14 \\
\hline & ICBF & 16 & 11.56 & (1000 & $050 * t+2 \div$ & 0 \\
\hline Laberıntos Planeacıon & Colegio & 11 & 17.55 & 49.000 & $.050 \times$ & -.38 \\
\hline & ICBF & 16 & 19.25 & 10 & 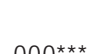 & 80 م \\
\hline 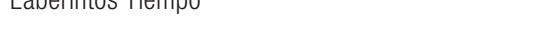 & Colegio & 11 & 6.36 & 4.000 & $.000 \mathrm{~m}$ & -.80 \\
\hline & ICBF & 16 & 12.44 & & & \\
\hline Clasifica de cartas Aciertos & Colegio & 11 & 16.27 & 63.000 & .216 & -.24 \\
\hline Clocifice do corton Dorcoworacione & ICBF & 16 & 18.00 & 240 & 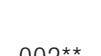 & \\
\hline Clasinca de cartas perseveraciones & Colegio & 11 & 8.18 & 24.000 & $.002^{\star n}$ & -.61 \\
\hline & ICBF & 16 & 18.19 & & & \\
\hline Clasifica de cartas Perseveraciones diferidas & Colegio & 11 & 7.91 & 21.000 & $.001^{\star \star}$ & -.65 \\
\hline & ICBF & 16 & 15.16 & & & \\
\hline Clasitica de cartas I Iempo & Colegio & 11 & 12.32 & 69.500 & .361 & -.18 \\
\hline
\end{tabular}


Continuación

\begin{tabular}{|c|c|c|c|c|c|c|}
\hline Variable & Grupo & $\mathrm{n}$ & Rango & $\mathrm{U}$ & $\mathrm{p}$ & $r$ \\
\hline \multirow{2}{*}{ Clasifica semántica Total categorías } & ICBF & 16 & 17.44 & \multirow{2}{*}{33.000} & \multirow{2}{*}{$.006^{\star \star}$} & \multirow{2}{*}{-.53} \\
\hline & Colegio & 11 & 9.00 & & & \\
\hline \multirow{2}{*}{ Clasifica semántica Promedio total animales } & ICBF & 16 & 15.00 & \multirow{2}{*}{72.000} & \multirow{2}{*}{.421} & \multirow{2}{*}{-.15} \\
\hline & Colegio & 11 & 12.55 & & & \\
\hline \multirow{2}{*}{ Clasifica semántica Puntaje total } & ICBF & 16 & 17.28 & \multirow{2}{*}{35.500} & \multirow{2}{*}{$.009 * *$} & \multirow{2}{*}{-.50} \\
\hline & Colegio & 11 & 9.23 & & & \\
\hline \multirow[b]{2}{*}{ Fluidez verbal Aciertos } & ICBF & 16 & 16.38 & \multirow[b]{2}{*}{50.000} & \multirow{2}{*}{.060} & \multirow[b]{2}{*}{-.36} \\
\hline & Colegio & 11 & 10.55 & & & \\
\hline \multirow[b]{2}{*}{ Fluidez verbal Perseveraciones } & ICBF & 16 & 15.03 & \multirow[b]{2}{*}{71.500} & \multirow[b]{2}{*}{.136} & \multirow[b]{2}{*}{-.29} \\
\hline & Colegio & 11 & 12.50 & & & \\
\hline \multirow{2}{*}{ Torre de Hanoi 3 discos Movimientos } & $\mathrm{ICBF}$ & 16 & 12.78 & \multirow{2}{*}{68.500} & \multirow{2}{*}{.333} & \multirow{2}{*}{-.19} \\
\hline & Colegio & 11 & 15.77 & & & \\
\hline \multirow{2}{*}{ Torre de Hanoi 3 discos Tiempo } & ICBF & 16 & 13.22 & \multirow{2}{*}{75.500} & \multirow{2}{*}{.537} & \multirow{2}{*}{-.12} \\
\hline & Colegio & 11 & 15.14 & & & \\
\hline \multirow{2}{*}{ Torre de Hanoi 4 discos Movimientos } & ICBF & 16 & 11.75 & \multirow{2}{*}{52.000} & \multirow{2}{*}{.460} & \multirow{2}{*}{-.15} \\
\hline & Colegio & 8 & 14.00 & & & \\
\hline \multirow{2}{*}{ Torre de Hanoi 4 discos Tiempo } & $\mathrm{ICBF}$ & 16 & 12.06 & \multirow{2}{*}{57.000} & \multirow{2}{*}{.668} & \multirow{2}{*}{-.09} \\
\hline & Colegio & 8 & 23.38 & & & \\
\hline
\end{tabular}

Nota. $n$ : Número de participantes; U: Resultado de la U de Mann Whitney; $p$ : Probabilidad; $r$ : Tamaño del efecto no paramétrico; ${ }^{*} p \leq .05 ;{ }^{* *} p \leq .01 ;{ }^{* \star *} p \leq .001$

Finalmente, en la Tabla 3 se relacionan los resultados de las comparaciones efectuadas con la $\mathrm{CBCL}$, que muestran que el GC obtuvo rangos de puntuaciones significativamente más altas en Problemas de Atención, con un tamaño del efecto moderado, mientras que las participantes del GA presentaron un rango de puntuaciones significativamente más alto en Rompimiento de Normas, con un tamaño del efecto moderado también. 
Tabla 3

Resultados de las comparaciones entre los grupos en las escalas de la CBCL.

\begin{tabular}{|c|c|c|c|c|c|c|}
\hline Variable & Grupo & $\mathrm{n}$ & Rango & U & $\mathrm{p}$ & $r$ \\
\hline \multirow{2}{*}{ Ansioso depresivo } & ICBF & 16 & 13.13 & \multirow{2}{*}{74.000} & \multirow{2}{*}{.488} & \multirow{2}{*}{-.13} \\
\hline & Colegio & 11 & 15.27 & & & \\
\hline \multirow{2}{*}{ Retraído depresivo } & ICBF & 16 & 13.44 & \multirow{2}{*}{79.000} & \multirow{2}{*}{.656} & \multirow{2}{*}{-.09} \\
\hline & Colegio & 11 & 14.82 & & & \\
\hline \multirow{2}{*}{ Quejas somáticas } & ICBF & 16 & 12.09 & \multirow{2}{*}{57.500} & \multirow{2}{*}{.130} & \multirow{2}{*}{$-.2 \varsigma$} \\
\hline & Colegio & 11 & 16.77 & & & \\
\hline \multirow{2}{*}{ Problemas sociales } & ICBF & 16 & 12.50 & \multirow{2}{*}{64.000} & \multirow{2}{*}{.234} & \multirow{2}{*}{-.23} \\
\hline & Colegio & 11 & 16.18 & & & \\
\hline \multirow{2}{*}{ Problemas de pensamiento } & ICBF & 16 & 13.53 & \multirow{2}{*}{80.500} & \multirow{2}{*}{.709} & \multirow{2}{*}{-.07} \\
\hline & Colegio & 11 & 14.68 & & & \\
\hline \multirow{2}{*}{ Problemas de atención } & ICBF & 16 & 11.16 & \multirow{2}{*}{42.500} & \multirow{2}{*}{$.024^{*}$} & \multirow{2}{*}{-.43} \\
\hline & Colegio & 11 & 18.14 & & & \\
\hline \multirow{2}{*}{ Rompimiento de normas } & ICBF & 16 & 16.50 & \multirow{2}{*}{48.000} & \multirow{2}{*}{$.047^{*}$} & \multirow{2}{*}{-.38} \\
\hline & Colegio & 11 & 10.36 & & & \\
\hline \multirow{2}{*}{ Conducta agresiva } & ICBF & 16 & 13.19 & \multirow{2}{*}{75.000} & \multirow{2}{*}{.520} & \multirow{2}{*}{-.12} \\
\hline & Colegio & 11 & 15.18 & & & \\
\hline \multirow{2}{*}{ Otros problemas } & ICBF & 16 & 13.28 & \multirow{2}{*}{76.500} & \multirow{2}{*}{.567} & \multirow{2}{*}{-.11} \\
\hline & Colegio & 11 & 15.05 & & & \\
\hline
\end{tabular}

Nota. n: Número de participantes; U: Resultado de la U de Mann Whitney; p: Probabilidad; r: Tamaño del efecto no paramétrico; ${ }^{*} \mathrm{p} \leq .05$

$\square$

\section{iscusión}

El objetivo general de esta investigación fue comparar el funcionamiento ejecutivo, conductas externalizantes e internalizantes de niñas y adolescentes entre los 8 y 15 años que vivían en un sector urbano de alta adversidad social en Bogotá, conocido como la "Zona del Bronx", con un grupo niñas y adolescentes con características sociodemográficas similares, vinculadas a un colegio distrital de la misma ciudad.
Los resultados sugieren que las participantes que habitaban dicha zona (GA) tenían menos estrategias para controlar la impulsividad y más conductas de rompimiento de normas, que las participantes del grupo de comparación, aunque presentaron rangos de puntuaciones más altos en FFEE como cambio, monitoreo, iniciativa, planificación/organización y memoria de trabajo visoespacial y en el índice global de función ejecutiva, como lo indican sus puntuaciones en las escalas de la BRIEF y las subpruebas de la BANFE 2. Las participantes del grupo de comparación, por su parte, presentaron puntuaciones más altas en problemas de atención. 
Estos resultados son coherentes con los de los estudios que muestran que las condiciones de adversidad social, por factores como la pobreza y la violencia, podrían favorecer el desarrollo de problemas de comportamiento infantil y adolescente (Barrasso-Catanzaro \& Eslinger, 2016; Troller et al., 2016), tales como los del estudio de Boutwell et al. (2017), en el que se encontró una relación entre problemas de comportamiento internalizante y externalizante y el Cl, entre 2591 niños estadounidenses cuyas familias estaban siendo investigadas por denuncias de maltrato y la investigación de Kersten et al. (2017), en la que se evidenció una relación estadísticamente significativa entre problemas de comportamiento y ser testigo de violencia en la comunidad, entre 1176 niños y adolescentes estadounidenses. También son consistentes con los resultados de los estudios realizados en Bogotá (Colombia), con niños, niñas y adolescentes que vivían en barrios económicamente desfavorecidos, quienes evidenciaron más problemas de conducta internalizante y/o externalizante (Hewitt, 2015; Moreno et al., 2014). Los actos de ilegalidad y la situación de desventaja social, comunes en la "Zona del Bronx", así como la falta de un control parental constante, según refiere Orozco (2016), podrían explicar el mayor número de actos de rompimiento de normas entre las participantes del GA.

No obstante, las participantes del colegio puntuaron más alto en problemas de atención que las que vivían en el Bronx, lo que sugiere que estas últimas requerían una mayor capacidad atencional. Es posible que ello se deba a la necesidad de estar alerta en un ambiente peligroso y estresante como lo fue la Zona del Bronx, aunque se requieren más estudios que examinen esta relación.

Los resultados también señalan que las conductas de rompimiento de normas, consideradas de tipo externalizante, también podrían presentarse frecuentemente entre las mujeres en entornos de alta adversidad social, en contraposición con los resultados de los estudios que indican que las mujeres presentan más conductas internalizantes y los hombres más conductas externalizantes, como el de Alarcón y Bárrig (2015).

Por otra parte, los resultados obtenidos con las pruebas de FFEE son inconsistentes, en general, con los de estudios que indican que los niños, niñas y adolescentes que crecen en entornos adversos, presentan puntajes más bajos en medidas de funcionamiento ejecutivo (Barrasso-Catanzaro \& Eslinger, 2016; Dike, 2017; Hostinar et al., 2012; Mani et al., 2013), como el estudio de Andrés et al. (2010), quienes encontraron un desempeño más bajo en FFEE entre los niños que asistían a escuelas públicas y obtuvieron una puntuación alta en conductas antisociales. En nuestra investigación, las participantes que vivían en la "Zona del Bronx", puntuaron más alto en cambio, monitoreo, iniciativa, planificación/organización, memoria de trabajo visoespacial y en el índice global de función ejecutiva de la BRIEF, resultados que son consistentes con los de aquellos estudios que sugieren que las exigencias sociales y ambientales de los entornos de alta adversidad social, como lo fue la zona del Bronx, exigen el desarrollo de este tipo de capacidades para sobrevivir, tales como los resultados del estudio de Mittal et al. (2015), quienes encontraron que los adultos que crecieron en ambientes violentos 
e impredecibles se desempeñaban mejor en la función ejecutiva de cambio, posiblemente porque este tipo de ambientes estimulan el desarrollo de esta habilidad para adaptarse rápidamente a los cambios imprevistos propios de este tipo de entornos.

No obstante, los resultados obtenidos en la prueba de ordenamiento alfabético de palabras de la BANFE 2, en el Ensayo 3 , señala que las participantes del GA presentaban una capacidad menor para manipular y ordenar mentalmente la información verbal contenida en la memoria de trabajo. Es posible que ello se deba a una menor estimulación y a una exigencia menor de la memoria de trabajo de material verbal entre las participantes que vivían en un entorno de alta adversidad social, comparadas con las del otro grupo, conformado por niñas y adolescentes que se encontraban escolarizadas. En similitud, Fernald, Weber, Galasso y Ratsifandrihamanana (2011) encontraron que los niños cuyas madres tenían educación secundaria se desempeñaron mejor en casi todas las medidas de desarrollo cognitivo y del lenguaje evaluadas y tuvieron un mejor crecimiento lineal, en comparación con los hijos de mujeres sin educación.

Por el contrario, en la subprueba de memoria de trabajo visoespacial, que estima la capacidad para retener y producir activamente el orden secuencial visoespacial de una serie de figuras, las participantes que vivían en el Bronx evidenciaron mejores puntuaciones, lo cual se puede explicar si se tiene en cuenta que un entorno como éste exige una gran habilidad para retener información de esa naturaleza, siendo ello coherente con la idea de que los entornos de alta adversidad social podrían moldear de manera adaptativa el funcionamiento ejecutivo y cognitivo desde temprana edad (Mittal et al., 2015). No obstante, se recomienda la realización de estudios similares que examinen el desempeño en pruebas de memoria de trabajo visoespacial entre niños, niñas y adolescentes expuestos a este tipo de entornos.

En la prueba de laberintos de la BANFE 2, por otra parte, las participantes que habitaban en el Bronx presentaron un mejor desempeño al resolver los laberintos con menos errores de planeación, aunque utilizaron un tiempo menor, lo que indica una mejor capacidad de planificación, como se encontró con el BRIEF, pero también una mayor tendencia a la impulsividad entre las participantes del Bronx, comparadas con las niñas y adolescentes del colegio. Este resultado es coherente con los del estudio de Quistberg y Mueller (2019), quienes encontraron que los errores en control inhibitorio se relacionaban con las conductas externalizantes en niños de 5 y 6 años de edad y el estudio de Madhushanthi et al. (2018), quienes informaron puntuaciones más bajas en memoria de trabajo e inhibición entre adolescentes mujeres de nivel socioeconómico bajo.

Es posible que esta impulsividad sea adaptativa en ambientes de alta adversidad social como el Bronx, ya que las recompensas futuras son más inciertas en este tipo de entornos que las recompensas inmediatas (Fawcett, McNamara \& Houston, 2012), pero se requieren más investigaciones sobre FFEE como el control inhibitorio, en niños, niñas y adolescentes expuestos a estos entornos.

Los resultados obtenidos con la subprueba de clasificaciones semánticas, 
señalan una mejor capacidad de abstracción, iniciativa y flexibilidad mental entre las participantes que vivían en el Bronx, ya que esta prueba evalúa la capacidad para analizar y agrupar en categorías semánticas una serie de figuras de animales con el mayor número posible de categorías. Estos resultados también son coherentes con los de algunas investigaciones que indican que los ambientes de alta adversidad social no necesariamente deterioran el funcionamiento ejecutivo, sino que lo moldean de manera adaptativa para responder a entornos altamente estresantes (Del Giudice et al., 2012; Ellis \& Del Giudice, 2014; Ellis et al., 2012; Frankenhuis \& Del Giudice, 2012; Nederhof \& Schmidt, 2012), pues este tipo de habilidades podrían ser adaptativas en contextos sociales adversos como lo fue la zona del Bronx, en donde era necesario interactuar con diferentes tipos de personas, en circunstancias impredecibles y cambiantes.

En conclusión, los resultados obtenidos en esta investigación indican que las niñas y adolescentes que vivían en el Bronx no necesariamente presentaban déficits en FFEE e, incluso, podrían presentar un desarrollo más alto en algunas funciones claves para la supervivencia en este tipo de entornos, como la capacidad de cambio, planificación e iniciativa. En contraposición a los resultados de estudios como el de Andrés et al. (2015), los resultados de la presente investigación sugieren que la exposición a este tipo de entornos podría moldear el funcionamiento mental en la infancia y adolescencia de maneras adaptativas. Por lo tanto, la experiencia de vida en este tipo de entornos es un conocimiento que podría resultar útil al atender a niñas y adolescentes por parte de especialistas en áreas como la neuropsicología, la psicología clínica infantil y la neuropediatría.

Como fortalezas de esta investigación se puede resaltar el uso de pruebas neuropsicológicas válidas para la evaluación de las FFEE y las conductas externalizantes e internalizantes, así como la posibilidad de realizar una evaluación completa de dichas funciones, contrastando los resultados obtenidos, en una muestra de difícil acceso como lo eran las participantes que vivían en el Bronx. Entre las limitaciones de este estudio se pueden destacar el tamaño de la muestra y su selección no aleatoria, lo cual podría haber generado sesgos de selección, así como el hecho de que la mayoría de los instrumentos utilizados no han sido validados con muestras colombianas. Además, los resultados fueron obtenidos con participantes mujeres, por lo que se hace necesaria la inclusión de hombres en futuras investigaciones y la realización de comparaciones por sexo. Se sugiere también, en futuros estudios, examinar otras variables neuropsicológicas como la atención, la memoria y el lenguaje.

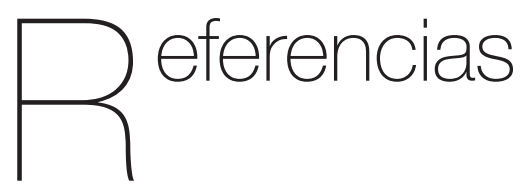

Achenbach, T.M. (1991). Manual for the CBCL/4-18 and 1991 profile. Burlington, VT: University of Vermont Department of Psychiatry.

Achenbach, T. M., \& Edelbrock, C. S. (1992). Child Behavior Checklist. Burlington, VT: University Associates in Psychiatric. 
Alarcón, D., \& Bárrig, P. (2015). Conductas internalizantes y externalizantes en adolescentes. Liberabit, 21(2), 253-259.

Andrés, M., Canet, L., García, A., Rubiales, J., Introzzi, I., \& Urquijo, S. (Noviembre, 2010). Desempeño ejecutivo en niños con conductas antisociales. Ponencia presentada en el IX Congreso Argentino de Neuropsicología, Buenos Aires.

Barrasso, C. C., \& Eslinger, P. J. (2016). Neurobiological bases of executive function and social-emotional development: Typical and atypical brain changes. Family Relations, 65(1), 108-119. doi: 2147/10.1111/ fare.12175

Boutwell, B. B., Helton, J., Vaughn, M. G., \& Kavish, N. (2017). The association of externalizing and internalizing problems with indicators of intelligence in a sample of atrisk children. Biorxiv. doi: 10.1101/210500.

Bravo, C. (2015). La producción de marginalidad urbana: el proceso sociohistórico, emergencia y configuración del Bronx en Bogotá (Tesis de grado). Universidad Santo Tomás, Bogotá, Colombia.

Cabas, K., Gonzalez, Y., \& Mendoza, C. (2018). Funcionamiento ejecutivo y depresión en universitarios con normopeso, sobrepeso y obesidad Tipo I. Informes Psicológicos, 18(1), 133-144. doi: 10.18566/infpsic. v18n1a07

Del Giudice, M., Hinnant, J. B., Ellis, B. J., \& El-Sheikh, M. (2012). Adaptive patterns of stress responsivity: A preliminary investigation. Developmental Psychology, 48, 775-790. doi: 10.1037/a0026519

Dike, V. E. (2017). Poverty and brain development in children: Implications for learning. Asian Journal of Education and Training, 3(1), 64-68. doi: 10.20448/ journal.522.2017.31.64.68.

Ellis, B. J., \& Del Giudice, M. (2014). Beyond allostatic load: Rethinking the role of stress in regulating human development. Development and Psychopathology, 26, 1-20. doi: 10.1017/S095457 9413000849

Ellis, B., Del Giudice, M., Dishion, T., Figueredo, A., Gray, P., Griskevicius, V., Hawley, P. H., Jacobs. W. J., James, J., Volk, A. A., \& Wilson, D. (2012). The evolutionary basis of risky adolescent behavior: Implications for science, policy, and practice. Developmental Psychology, 48, 598-623. doi: 10.1037/ a0026220

Fawcett, T. W., McNamara, J. M., \& Houston, A. I. (2012). When is it adaptive to be patient? A general framework for evaluating delayed rewards. Behavioural Processes, 89, 128136. doi: 10.1016/j.beproc.2011.08.015

Fernald, L. C., Weber, A., Galasso, E., \& Ratsifandrihamanana, L. (2011). Socioeconomic gradients and child development in a very low income population: evidence from Madagascar. Developmental Science, 14(4), 832-847.

Flores, J., \& Ostrosky-Shejet, F. (2012). Desarrollo neuropsicológico de lóbulos frontales y funciones ejecutivas. México: Manual Moderno.

Flores, J., Ostrosky, F., \& Lozano, A. (2014). Batería Neuropsicológica de las Funciones Ejecutivas y Lóbulos Frontales. México: Manual Moderno.

Flores-Lázaro, J., Castillo-Preciado, R., \& Jiménez-Miramonte, N. (2014). Desarrollo de funciones ejecutivas, de la niñez a la 
juventud. Anales de Psicología, 30(2), 463-473.

Frankenhuis, W. E., \& Del Giudice, M. (2012). When do adaptive developmental mechanisms yield maladaptive outcomes? Developmental Psychology, 48, 628-642. http://dx.doi.org/10.1037/a0025629

Gioia, G., Isquith, P., Guy, S., \& Kenworthy, L. (2000). Behavior Rating Inventory of Executive Function: BRIEF. Odessa, FL: Psychological Assessment Resources.

Hatoum, A. S., Rhee, S. H., Corley, R. P., Hewitt, J. K., \& Friedman, N. P. (2018). Do executive functions explain the covariance between internalizing and externalizing behaviors? Development \& Psychopathology, 30(4), 1371-1387. doi: 10.1017/S0954579417001602

Hewitt, N. (2015). Predicción de los problemas de banda ancha y banda estrecha mduenta la integración de los modelos transaccional, cognitivo-social y de regulación emocional (Tesis doctoral). Universidad de Granada, España.

Hewitt, N., Jaimes, S., Vera, L., \& Villa, M. (2012). Características psicométricas del cuestionario de comportamientos infantiles CBCL en niñosyadolescentes colombianos (Tesis de pregrado). Universidad de San Buenaventura Bogotá.

Hostinar, C., Stellern, S., Schaefer, C., Carlson, S., \& Gunnar, M. (2012). Associations between early life adversity and executive function in children adopted internationally from orphanages. Proceedings of the National Academy of Sciences of the United States of America, 109, 1720817212. doi: 10.1073/pnas.1121246109
Kaufman, A. S., \& Kaufman, N. L. (2000). K-BIT: Test breve de inteligencia de Kaufman. Madrid: TEA.

Kersten, L., Vriends, N., Steppan, M., Raschle, N. M.., Praetzlich, M., Oldenhof, H., et al. (2017). Community violence exposure and conduct problems in children and adolescents with conduct disorder and healthy controls. Frontiers in Behavioral Neuroscience, 11, 1-14. doi: 10.3389/ fnbeh.2017.00219.

Lezama, E. (2015). Relación de las funciones ejecutivas con trastornos externalizantes y/o internalizantes en niños de 5 a 15 años con TDAH que asisten a programas de atención a la población infantil de la ciudad de Manizales (Tesis de Maestría). Universidad de Manizales, Manizales, Colombia.

Madhushanthi, H. J., Wimalasekera, S. W., Goonewardena, C. S. E., Amarasekara, A. A. T. D., \& Lenora, J. (2018). Socioeconomic status is a predictor of neurocognitive performance of early female adolescents. International Journal of Adolescent Medicine and Health. doi: 2147/10.1515/ ijamh-2018-0024

Mani, A., Mullainathan, S., Shafir, E., \& Zhao, J. (2013). Poverty impedes cognitive function. Science, 341, 976-980. http://dx.doi. org/10.1126/ science.1238041.

Mittal, C., Griskevicius, V., Simpson, J., Sung, S., \& Young, E. (2015). Cognitive adaptations to stressful environments: When childhood adversity enhances adult executive function. Journal of Personality and Social Psychology, 109(4), 604-621.

Moreno, J., Echavarría, K., Pardo, A., \& Quiñones, Y. (2014). Funcionalidad familiar, 
conductas internalizadas y rendimiento académico en un grupo de adolescentes de la ciudad de Bogotá. Psychologia: Avances de la Disciplina, 8(2), 37-46.

Moreno, J., \& Utria, E. (2011). Caracterización de los programas de tratamiento cognitivoconductual para el manejo de problemas de comportamiento en niños y adolescentes realizados en Bogotá entre 2002 y 2008. Psicología Desde el Caribe, (28), 39-76.

Nederhof, E., \& Schmidt, M. (2012). Mismatch or cumulative stress: Toward an integrated hypothesis of programming effects. Physiology \& Behavior, 106, 691-700. doi: 10.1016/j.physbeh.2011.12.008.

Orozco, C. (4 de junio de 2016). Los niños del Bronx, la realidad más trágica. El Espectador. Recuperado de http://www. elespectador.com/entrevista-de-ceciliaorozco/los-ninos-del-bronx-realidad-mastragica-articulo-635972.

Quistberg, K. A., \& Mueller, U. (2019). Prospective relations between kindergarteners' executive function skills and their externalizing and internalizing behaviors. The Clinical Neuropsychologist, 1-18. doi: 10.1080/13854046.2019.1591510

Rodríguez-Sabiote, C. (2016). Metodología de Investigación en Ciencias Humanas y Sociales. En J. F. Soares (Org.), Discussões epistemológicas: as Ciências Humanas sob uma ótica interdisciplinar (pp. 3560). São Luís: Universidade Federal do Maranhão.

Tirapu, J., García, A., Luna, P., Verdejo, A., \& Ríos. M. (2012). Corteza prefrontal, funciones ejecutivas y regulación de la conducta. En J. Tirapu, A. García, M. Ríos y A. Ardila (Eds), Neuropsicología de la corteza prefrontal y las funciones ejecutivas (pp. 87-117). Barcelona: Viguera.

Tirapu-Ustárroz, J., García-Molina, A., LunaLario, P., Roig-Rovira, T., \& Pelegrín-Valero, C. (2008). Modelos de funciones y control ejecutivo (II). Revista de Neurología, 46(12), 742-750.

Troller, R. S., Nelson, C. A., Zeanah, C. H., \& Fox, N. A. (2016). Deficits in error monitoring are associated with externalizing but not internalizing behaviors among children with a history of institutionalization. Journal of Child Psychology \& Psychiatry, 57(10), 1145-1153. doi: 10.1111/jcpp.12604

Woltering, S., Lishak, V., Hodgson, N., Granic, I., \& Zelazo, P. D. (2016). Executive function in children with externalizing and comorbid internalizing behavior problems. Journal of Child Psychology \& Psychiatry, 57(1), 3038. doi: 10.1111/jcpp.12428 